\title{
Measuring the Spatial Frequency Transfer Function of Phase Measuring Interferometers for Laser Optics
}

C. R. Wolfe

J. D. Downie

J. K. Lawson
RECEIVED

AUG 1619

OSTI

This paper was prepared for submittal to the SPIE 3rd International Workshop on Laser Beam and Optics Characterization

Quebec, Canada

July 8-10, 1996

June 27, 1996

This is a preprint of a paper intended for publication in a journal or proceedings. Since changes may be made before publication, this preprint is made available with the understanding that it will not be cited or reproduced without the permission of the author.

DISTRIBUTTON OF THIS DOCUMENT IS UNLMMTED 


\section{DISCLAIMER}

This document was prepared as an account of work sponsored by an agency of the United States Government. Neither the United States Government nor the University of California nor any of their employees, makes any warranty, express or implied, or assumes any legal liability or responsibility for the accuracy, completeness, or usefulness of any information, apparatus, product, or process disclosed, or represents that its use would not infringe privately owned rights. Reference herein to any specific commercial product, process, or service by trade name, trademark, manufacturer, or otherwise, does not necessarily constitute or imply its endorsement, recommendation, or favoring by the United States Government or the University of California. The views and opinions of authors expressed herein do not necessarily state or reflect those of the United States Government or the University of California, and shall not be used for advertising or product endorsement purposes. 


\section{DISCLAIMER}

Portions of this document may be illegible in electronic image products. Images are produced from the best available original document. 


\title{
Measuring the spatial frequency transfer function of phase measuring interferometers for laser optics
}

\author{
C. R. Wolfe, J. D. Downie and J. K. Lawson \\ Lawrence Livermore National Laboratory, L-493 \\ P.O. Box 808 \\ Livermore, CA 94551 \\ wolfe2@llnl.gov \\ $510-422-3516$ (voice) \\ 510-423-4606 (FAX)
}

\begin{abstract}
The power spectral density function (PSD) is being employed to specify the surface finish and transmitted wavefront in the mid-spatial frequency regime for laser beam optics of the National Ignition Facility (NIF). The instrument used to measure the PSD is a phase measuring Fizeau interferometer. The phase map produced by the interferometer is digitally processed to create the PSD. Before one can use the PSD information, it is necessary to evaluate the fidelity of the interferometer spatial frequency response. Specifically, one must measure the overall transfer function of the instrument. To accomplish this, we perform a two-step "calibration" process. We first measure a known precision phase object with the interferometer and then compare the measured PSD to an ideal numerical simulation which represents the theoretical PSD. The square root of the ratio of the measured function to the simulation is defined as the transfer function of the instrument. We present experimental results for both reflective and transmissive test objects, including effects such as the test object orientation and longitudinal location in the interferometer cavity. We also evaluate the accuracy levels obtained using different test objects.
\end{abstract}

\section{Introduction}

This paper describes work completed in support of optical component development and laser system modeling for the National Ignition Facility (NIF). The NIF is sponsored by the U.S. Department of Energy, and design work is being conducted by several DOE laboratories and subcontractors within the National Inertial Confinement Fusion Program. The NIF will be an experimental fusion facility which will include a laser and a target area. The NIF laser will be capable of producing 500 TW at a wavelength of $0.35 \mu \mathrm{m}$ with specific symmetry, beam balance, and pulse shape. 
The performance of high-peak power laser systems, such as the NIF, can be seriously degraded by the presence of modulations in the beam. Beam degradation can result in energy loss on target and, in extreme circumstances, damage of optical components. Beam modulations are introduced or "imprinted" on the beam as it propagates by interaction with optical components. Examples of noise sources encountered by the beam include:

i. diffraction caused by hard edges and isolated defects within the clear aperture

ii. variation in index of refraction and/or stress birefringence of transmissive optical materials

iii. modulation in the contour of surfaces

iv. variation of phase retardation or reflectivity of optical coatings.

The sensitivity or tolerance of the laser to beam modulation is determined by the subsequent growth (amplification) of these modulations. This is dictated by the physics of non-linear optical gain and the geometry of spatial filtering employed for beam smoothing. The characteristic scale length of modulations that undergo amplification ranges from about 0.12 to $33 \mathrm{~mm}$. These features are commonly referred to as mid-spatial scale length modulations to distinguish them from simple roughness (scale lengths $<0.12 \mathrm{~mm}$ ) and figure errors (scale lengths $>33 \mathrm{~mm}$ ). ${ }^{1,2}$

This paper describes techniques and measurements to characterize the spatial frequency response of interferometric measurements. Modern phase measuring interferometers can record an entire phase map corresponding to an optical wavefront. These instruments use phase measuring techniques and high-density solid-state detectors to calculate the optical phase at each pixel in the wavefront. Interferometers using standard video detectors (e.g., $640 \times 480$ pixels class cameras) produce wavefront phase maps containing $>300,000$ data points, depending on the aperture measured. Instruments with higher density detectors (e.g., $1024 \times 1024$ pixel class cameras) are currently being developed. With such wavefront data collection capability, the fidelity of the spatial frequency response of the interferometer becomes an important figure of merit for the instrument. Interpretation of the spatial frequency content of the data requires that the over-all frequency dependent transfer function, $G(v)$, of the interferometer be determined. The transfer function determines the bandwidth of the instrument; thus, it dictates the number and type of measurements required to accurately measure the spatial frequency content in a wavefront.

\section{Fourier wavefront analysis/interferometer frequency response}

In order to deal directly with amplification of beam modulations in highpeak-power laser systems, we employ 2-D Fourier analysis ${ }^{3,4}$ to characterize 
the wavefront. We express the wavefront phase map as a discrete 2-D Fourier series. The low- and high-frequency limits of the series are determined by the test aperture dimension, (L), and number of pixels contained in the detector, $(\mathrm{N})$. Generally in our work, we define the data valid range as:

$$
\begin{gathered}
v_{\text {low }}=3 / L \text { and } \\
v_{\text {high }}=N / 4 L
\end{gathered}
$$

The 2-D power spectral density, $\operatorname{PSD}\left(v_{x}, v_{y}\right)$, of the wavefront is then calculated from the squares of the Fourier coefficients. The $\operatorname{PSD}\left(v_{x}, v_{y}\right)$ is subsequently used extensively in our laser system modeling and simulation work because it quantifies the amount and direction of scattered light. To first order, the distribution of light at the focal spot of the system is the summation of the $\operatorname{PSD}\left(v_{x}, v_{y}\right) s$ of the system components. ${ }^{5}$ Non-linear amplification occurs at certain frequencies, several references to these non-linear phenomona can be found in (4).

When used to specify optical wavefront quality, the 2-D PSD is usually collapsed into a 1-D representation, $\operatorname{PSD}(v)$, by summing over the direction ortogonal to the direction of interest:

$$
\operatorname{PSD}\left(v_{x}\right)=\Sigma_{v_{1}} v_{2} \operatorname{PSD}\left(v_{x}, v_{y}\right) \Delta v_{y^{*}}
$$

This approach is only justified if the direction of summation is perpendicular to the peak modulation in the wavefront or if the modulation is isotropic (e.g., there is no directionality). The result is equivalent to the average of the PSDs computed from all possible 1-D traces taken in the direction of interest.

Fourier representation of the wavefront and subsequent calculation of the power spectral density does not account for the spatial frequency response of the interferometer. This frequency response is given by the transfer function, $G(v)$, of the instrument. In general, the transfer function is approximately one at low frequencies and decreases as the high frequency (Nyquist) limit of the instrument is approached. The transfer function contains contributions from the interferometer optical system and detector, ${ }^{6}$ signal processing electronics and software algorithms. These sub-systems are complex and, in many instances, proprietary; their contributions to the transfer function are difficult to calculate separately. In addition, factors external to the interferometer, such as the operating environment of the instrument and physical configuration used for the measurement, also contribute to the transfer function. Because of these facts, we have chosen to determine the transfer function experimentally. We are determining the $G(v)$ of the interferometers we use for optical component characterization in the 
environments and configurations used to make those measurements. To do this, we compare the power spectral density of the measured wavefront from a known phase object with the theoretical PSD for that object.

\section{Measurement / calculation of the transfer fumction}

Two types of test phase objects have been fabricated and used successfully for this work. Both are sharp step-phase objects, first suggested and fabricated by Takacs. ${ }^{7}$ They have a simple Fourier spectrum with coefficients proportional to the step height and inversely proportional to the spatial frequency, $v$.

The phase object used for measurement of surface contour (or reflected wavefront) was anisotropically etched on the polished surface of a $101 \mathrm{~mm}$ diameter $\times 1 \mathrm{~mm}$ thick wafer of single crystal silicon. ${ }^{8}$ The phase object used for measurement of transmitted wavefront was etched on one surface of a $50 \mathrm{~mm}$ diameter $\times 10 \mathrm{~mm}$ thick fused silica substrate. ${ }^{9}$ The measured phase maps of these objects are shown in Figures 1a and 2. The reflected wavefront (Figure 1a) has a square aperture measuring approximately $70 \times 70 \mathrm{~mm}$. The wavefront contains, in addition to the sharp step, approximately 20 waves $(\lambda=633 \mathrm{~nm})$ of long scale length curvature. This is primarily optical power due to static convex deformation of the thin wafer. The transmitted wavefront (Figure 2) has a square aperture measuring approximately $40 \times 40 \mathrm{~mm}$. This wavefront contains, in addition to the sharp step, approximately 0.16 wave of long scale length curvature. This is due to nonflat and nonparallel surfaces of the substrate.

Ideal simulations of these sharp phase steps are used to calculate the transfer function from the measured wavefronts. These simulations are produced by creating numerical files (tables) containing the same number of data points as there are pixels in the measured data. The aperture dimensions are scaled to equal the measured phase maps. These simulated wavefronts are perfectly flat and contain only the sharp step transition. The height of the step is the same as the etched steps in the phase objects. The file created to simulate the reflected wavefront phase step is shown in Figure 1b. Using files such as these, the transfer function of the interferometer can be calculated. In each case, the result is unique to the instrument, the type of measurement (e.g., for reflected or transmitted wavefront measurements), aperture size, camera magnification, and other operational variables.

To simplify the presentation of the transfer function calculation, the 1-D representation of the $\operatorname{PSD}(v)$ described above, is used. First, the $\operatorname{PSD}\left(v_{x}, v_{y}\right)$ is calculated for both the measured wavefront and the corresponding simulated wavefront. The direction perpendicular to the step is determined in the wavefronts, and the PSD is collapsed to 1-D, $\operatorname{PSD}(v)$ along that direction. The 
long scale length (low frequency) waviness present in the measured wavefront is removed by performing a logical operation:

$$
\begin{aligned}
& \text { if, }\left(\operatorname{PSD}(v)_{\text {measured }}>\operatorname{PSD}(v)_{\text {simulated }}\right. \\
& \text { then, } G(v)=1 \text {. }
\end{aligned}
$$

Otherwise, the transfer function is determined from the ratio of the $\operatorname{PSD}(v)$ of the measured wavefront to the $\operatorname{PSD}(v)$ of the simulated wavfront:

$$
\mathrm{G}(v)=\left[\operatorname{PSD}(v)_{\text {measured }} / \operatorname{PSD}(v)_{\text {simulation }}\right]^{0.5} \text {. }
$$

Most interferometers are equipped with "pixelated" solid state detectors. For these instruments, the transfer function is 2-D (e.g., the $\left.G=G\left(v_{x}, v_{y}\right)\right)$. Here, for simplicity, we present only the 1-D result. The phase objects used in calibration are carefully oriented parallel to the pixel grid of the detector. The effect of "misalignment" is handled in a straight-forward manner when the full 2-D PSD is used to calculate the transfer function. This work is in progress and will be reported in the future.

The transfer function, $G(v)$, perpendicular to the step direction for reflected and transmitted wavefront measurements are shown in Figures 3 and 4.

Measurement of reflected wavefront from a small area $(10 \times 7.5 \mathrm{~mm})$ of the etched silicon step described above have been made. For this purpose, a beam-reducing telescope was attached to a commercial phase-measuring Fizeau interferometer. The transfer function is shown in Figure 5.

\section{Other calibration objects}

While straight-forward to fabricate, there are two disadvantages of the sharp step phase object. The first disadvantage is that the Fourier series or equally, the PSD, from a sharp step is undefined at the origin (zero frequency). The second disadvantage is that the signal-to-noise ratio is relatively low at high frequency since the Fourier coefficients are small in this range.

Other phase objects can potentially remove these difficulties. An ideal "tophat" phase object has a Fourier spectrum of a "sinc" function and a $\operatorname{PSD}(v)$ that is a sinc. ${ }^{2}$ The sinc and sinc functions are equal to one at zero frequency. However, to date, we have found it difficult computationally to handle the zeros that occur at certain frequencies in the sinc and sinc ${ }^{2}$ functions. One solution is to make the "tophat" object very narrow (on the order of $10-30 \mu \mathrm{m}$ wide), thus moving the zeros in the PSD to high (and 
uninteresting) frequencies. The resulting phase objects can only be measured at high magnification (e.g., using interference microscopy) since the return signal is very small. They have no practical application to mid-spatial scale length measurements.

A "white noise" phase object ${ }^{10}$ has the potential for providing consistently high signal-to-noise at all frequencies of interest. While less straight-forward to fabricate than the sharp step or the "tophat" phase objects, we believe it will be possible to approximate one using our kinoform phase plate technology developed at Livermore and at the Laboratory for Laser Energetics at University of Rochester. ${ }^{11}$

\section{Conclusions}

We have demonstrated a two-step method to calculate the spatial frequency transfer function of an interferometer by measuring a known phase object. The transfer function is unique to each test set-up; it must be determined for each test configuration. Experimental variables, such as cavity length, test component location, and working distance, all contribute, along with the physical principles of diffraction, to effect image formation at the detector.

With this information, it becomes possible to restore high spatial frequency information lost in the measurement of optical components. This technique is well known in Fourier analysis (restoration filtering) but has not been applied as an optimization tool, to our knowledge, in laser system design. The restored wavefront data is given by:

$$
\operatorname{PSD}(v)_{\text {restored }}=\operatorname{PSD}(v)_{\text {measured }} / \mathrm{G}(v)^{2} \text {. }
$$

We are continuing this work on several fronts:

i. to characterize the $G(v)$ for practical test configurations for the NIF Project

ii. to develop new calibration techniques and novel phase objects

iii. to guide the development of advanced interferometric test instruments. 


\section{Acknowledgments}

The authors wish to acknowledge co-workers at the Lawrence Livermore National Laboratory who have contributed to this work. F. DeMarco and D. Pigg performed many of the interferometric measurements of optical components. D. Ciarlo and Dr. I. M. Thomas fabricated the calibrated phase objects discussed here. This work was performed under the auspices of the U.S. Department of Energy by Lawrence Livermore National Laboratory under Contract W-7405-Eng-48.

\section{References}

1. D. M. Aikens, "The origin and evolution of optics specifications for the National Ignition Facility," Proc. SPIE, Vol. 2536, p. 2, 1995.

2. C. R. Wolfe et al., "Measurement of wavefront structure from large aperture optical components by phase shifting interferometry," Proc. SPIE, Vol. 2536, p. 13, 1995.

3. J. K. Lawson et al., "Specification of optical components using the power spectral density function," Proc SPIE, Vol. 2536, p. 38, 1995.

4. J. K. Lawson et al., "Power spectral density specifications for high power laser systems," in press Proc SPIE - Optical Systems Design and Production II, Glasgow, Scotland, May 1996.

5. J. E. Harvey et al., "Scattering effects from residual optical fabrication errors," Proc. SPIE, Vol. 2576, p. 25, 1995.

6. E. L. Church et al., "Direct comparison of mechanical and optical measurements of the finish of precision machined optical surfaces," Optical Engineering, Vol. 24, p. 3, 1985.

7. P. Z. Takacs, "A step height standard for surface profile characterization," Proc. SPIE, Vol. 1993, p. 65, 1993.

8. Fabricated by D. R. Ciarlo, Microstructures Laboratory, LLNL.

9. Fabricated by I. M. Thomas, Laser Program, LLNL.

10. A. Daniels et al., "Random transparency targets for modulation transfer function measurements in the visible and intrared regions," Opt. Eng., Vol. 34, p. 860, 1995.

11. S. N. Dixit et al., "Continous contour phase plates for tailoring the focal plane irradiance profile," Proc. SPIE, Vol. 2633, p. 141, 1995. 
Figure 1a:

Reflected wavefront from silicon wafer containing $101 \mathrm{~nm}$ step.

Test aperture is $70 \times 70 \mathrm{~mm}$ square, over-all P-V is approximately $20 \lambda$.

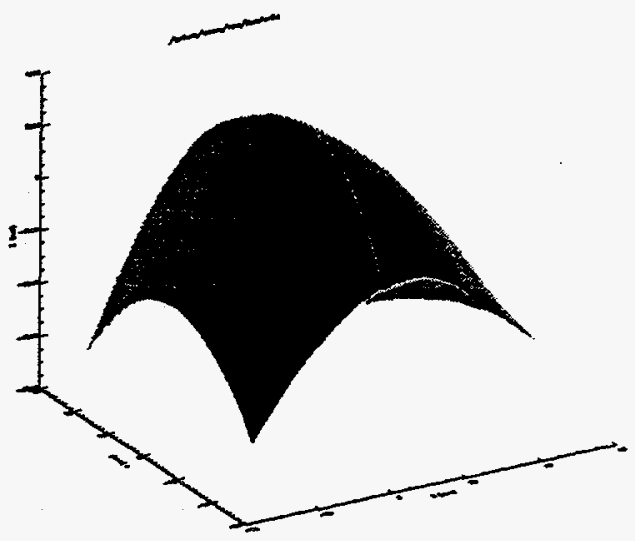

Figure 1b:

Simulation file used to calculate the interferometer transfer function for reflected wavefront measurement.

Test aperture $70 \times 70 \mathrm{~mm}$, step height $101 \mathrm{~nm}$.

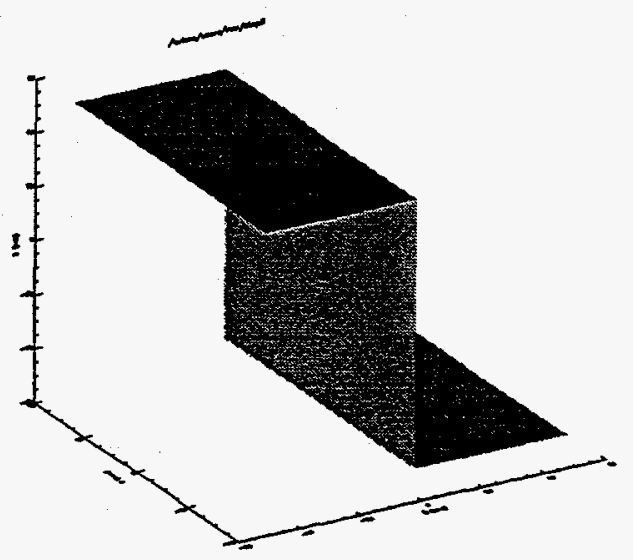

Figure 2:

Transmitted wavefront from fused silica substrate

Test aperture $40 \times 40 \mathrm{~mm}$, over-all P-V approximately $120 \mathrm{~nm}$.

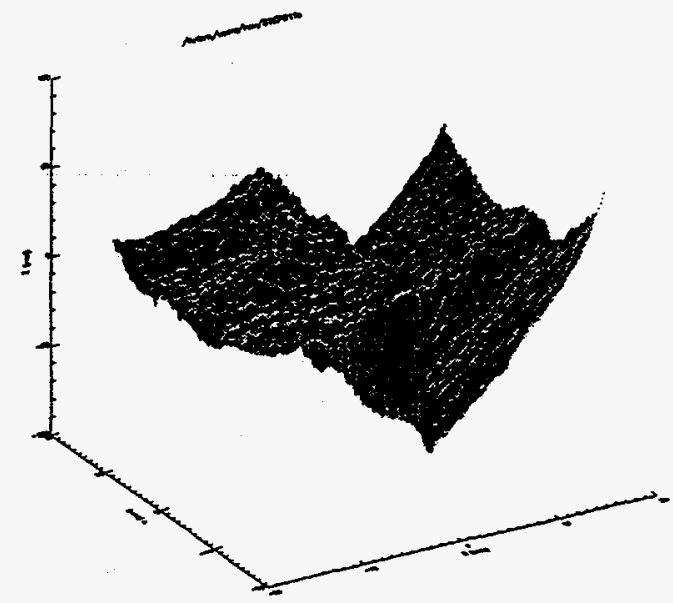




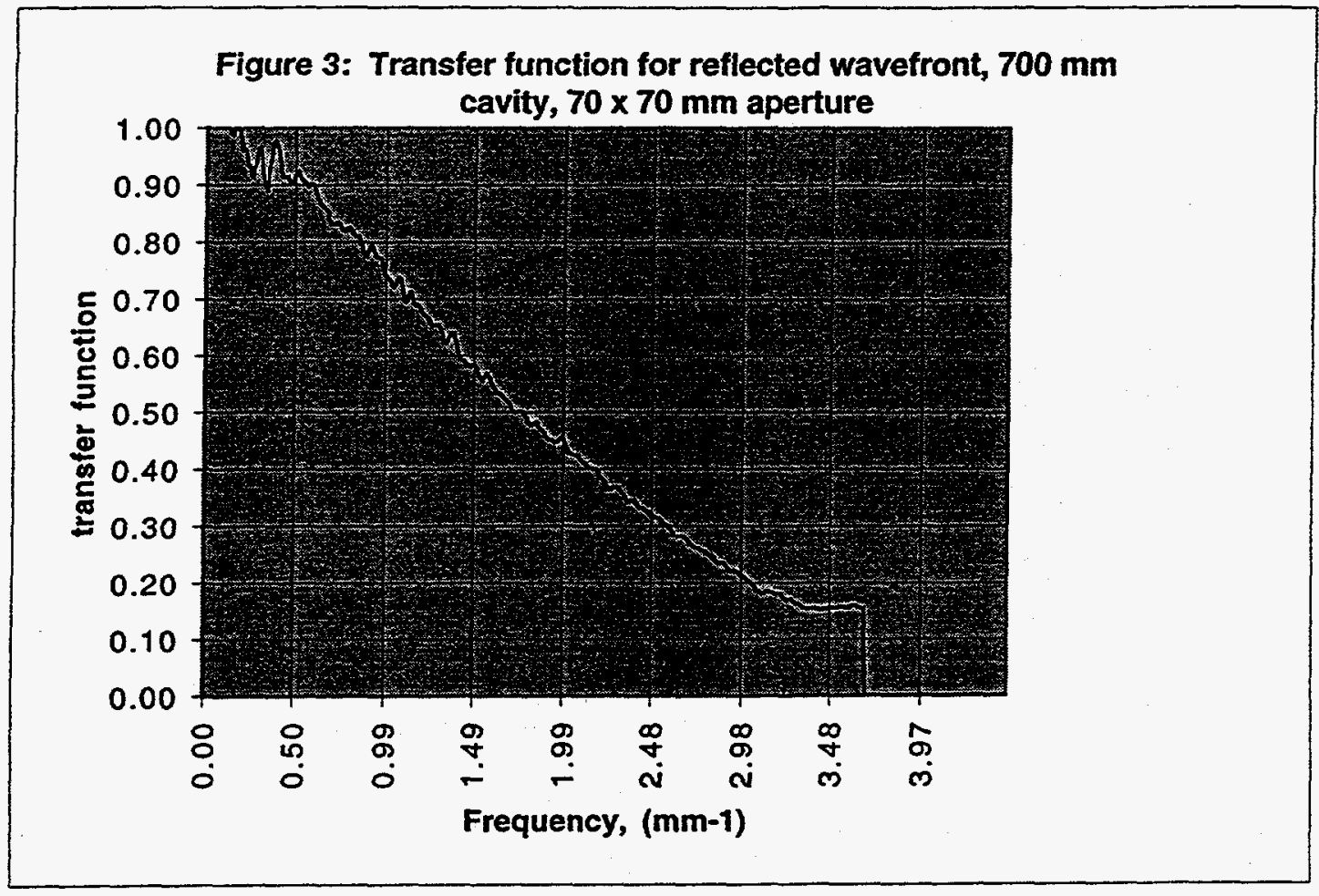

Figure 4: Transfer function for transmitted wavefront, $500 \mathrm{~mm}$ cavity, $\mathbf{4 0} \times \mathbf{4 0} \mathbf{~ m m}$ aperture

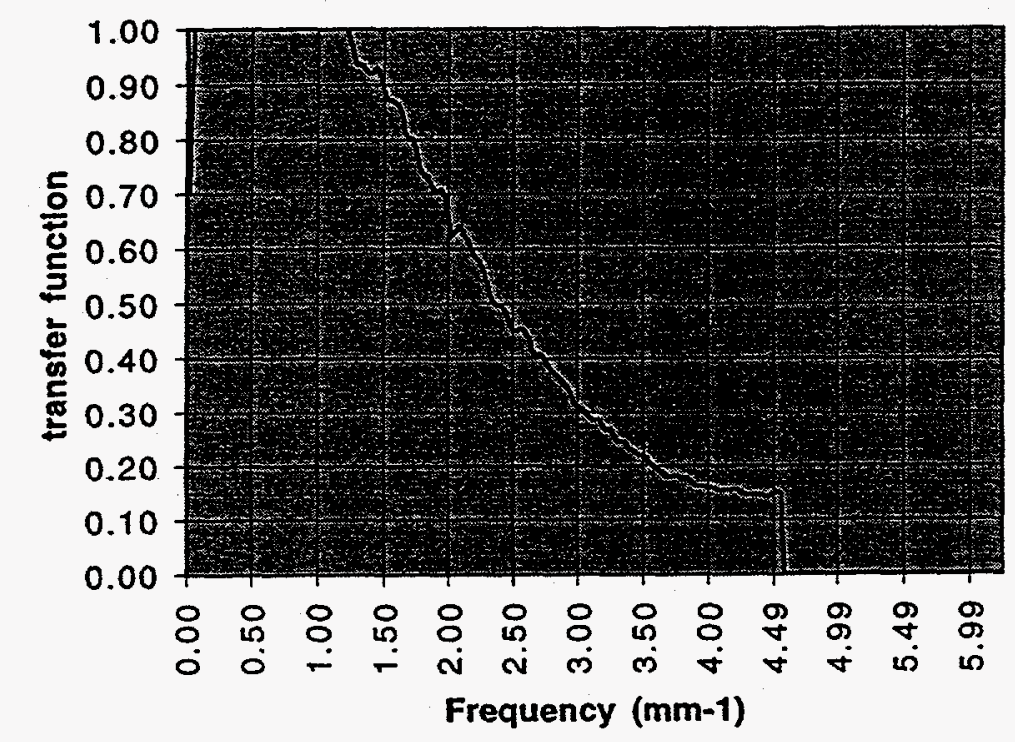


Figure 5: Tranfer function for reflected wavefront, $7.5 \times 10 \mathrm{~mm}$ aperture

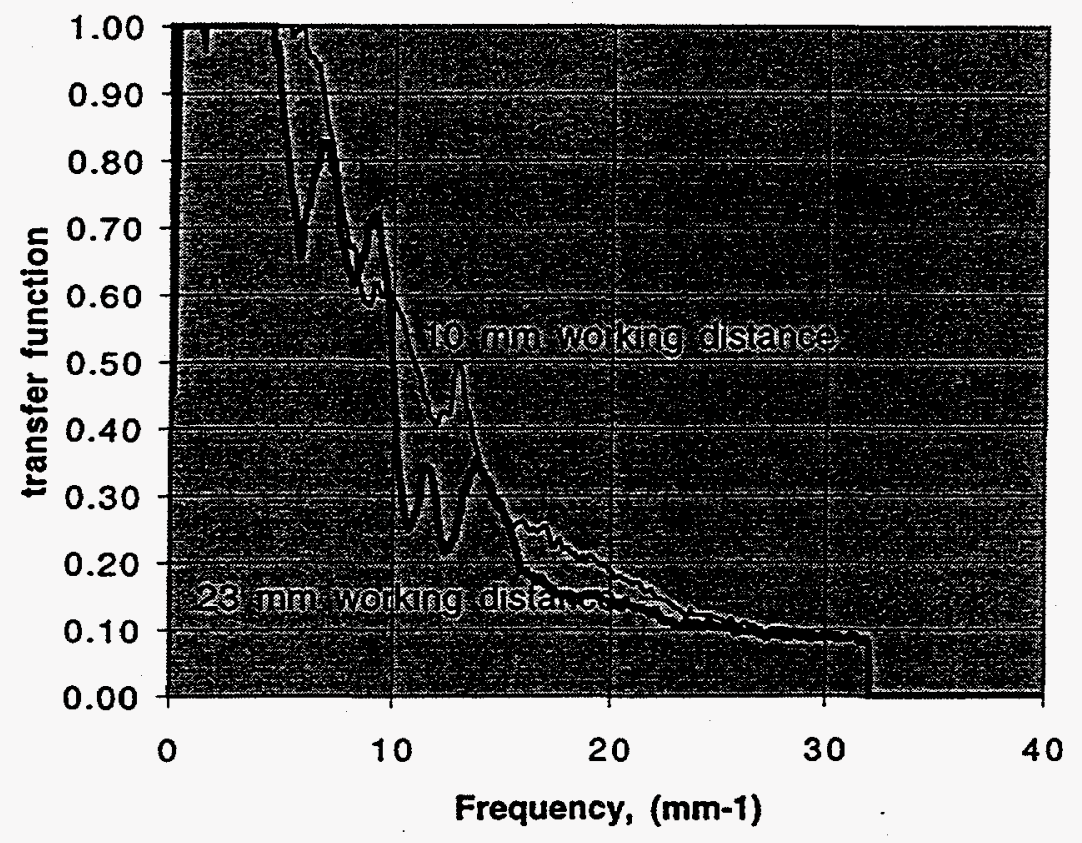

